\title{
Metabolomic Pathways Predicting Labor Dystocia by Maternal Body Mass Index
} \author{
Anne Dunlop, MD, MPH ${ }^{3}$ Dean Jones, $\mathrm{PhD}^{4}$ \\ ${ }^{1}$ Emory University Nell Hodgson Woodruff School of Nursing, Atlanta, \\ Georgia \\ 2 Department of Physiology, Columbia University School of Nursing, \\ New York, New York \\ ${ }^{3}$ Departments of Family and Preventive Medicine, Epidemiology, and \\ Nursing, Emory University, Atlanta, Georgia \\ ${ }^{4}$ Division of Pulmonary, Allergy, and Critical Care, Department of \\ Medicine, Emory University, Atlanta, Georgia
}

Nicole S. Carlson, PhD, CNM ${ }^{1}$ Jennifer K. Frediani, PhD, RD, ACSM-CES ${ }^{1}$ Elizabeth J. Corwin, PhD, RN²

Address for correspondence Nicole S. Carlson, PhD, CNM, Emory University Nell Hodgson Woodruff School of Nursing, 1520 Clifton Road NE, Atlanta, GA 30322 (e-mail: nicole.carlson@emory.edu).

Am J Perinatol Rep 2020;10:e68-e77.

\begin{abstract}
Keywords

- labor dystocia

- obesity

- mechanisms

- metabolomics

- parturition

Objectives The purpose of this study was to evaluate the metabolic pathways activated in the serum of African-American women during late pregnancy that predicted term labor dystocia.

Study Design Matched case-control study ( $n=97 ; 48$ cases of term labor dystocia and 49 normal labor progression controls) with selection based on body mass index (BMI) at hospital admission and maternal age. Late pregnancy serum samples were analyzed using ultra-high-resolution metabolomics. Differentially expressed metabolic features and pathways between cases experiencing term labor dystocia and normal labor controls were evaluated in the total sample, among women who were obese at the time of labor (BMI $\geq 30 \mathrm{~kg} / \mathrm{m} 2$ ), and among women who were not obese.

Results Labor dystocia was predicted by different metabolic pathways in late pregnancy serum among obese (androgen/estrogen biosynthesis) versus nonobese African-American women (fatty acid activation, steroid hormone biosynthesis, bile acid biosynthesis, glycosphingolipid metabolism). After adjusting for maternal BMI and age in the total sample, labor dystocia was predicted by tryptophan metabolic pathways in addition to C21 steroid hormone, glycosphingolipid, and androgen/estrogen metabolism.

Conclusion Metabolic pathways consistent with lipotoxicity, steroid hormone production, and tryptophan metabolism in late pregnancy serum were significantly associated with term labor dystocia in African-American women.
\end{abstract}

Cesarean birth increased steadily in the United States over the past several decades across all gestational ages, maternal ages, and racial groups. ${ }^{1-3}$ After a brief plateau from 2009 to 2016 , the cesarean delivery rate rose again in 2017 to reach $32.0 \%{ }^{4}$ Morbidity after primary cesarean and in subsequent pregnancies is increased due to wound complications, deep vein thrombosis and pulmonary embolism, bladder injury, obstet-

received

July 22, 2019

accepted after revision

December 20, 2019
DOI https://doi.org/

10.1055/s-0040-1702928. ISSN 2157-6998. rical hemorrhage, placenta previa/accreta, and cesarean hysterectomy. $^{5-7}$ For this reason, a key strategy identified by the Centers for Disease Control and Prevention to decrease pregnancy-related deaths is to avoid the use of cesarean delivery unless medically indicated. ${ }^{8}$

Cesarean deliveries are most often performed for abnormally slow labor progression, a complication known as labor
Copyright $\odot 2020$ by Thieme Medical Publishers, Inc., 333 Seventh Avenue, New York, NY 10001, USA. Tel: +1(212) 760-0888.
License terms

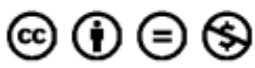


dystocia. $^{9,10}$ Expectations for labor progress are based on findings from large studies showing the median time required for changes in the uterine cervix during labor. ${ }^{11,12}$ Secondary analyses of these studies showed that there is a dose-dependent slowing of labor with elevations in maternal body mass index (BMI); for example, a woman having her first baby who has a $\mathrm{BMI} \geq 40 \mathrm{~kg} / \mathrm{m}^{2}$ may take up to 7 hours longer than a normal weight women to progress from 4 to $10 \mathrm{~cm}$ of cervical dilation. ${ }^{13}$ Largely due to labor dystocia, ${ }^{14}$ women with higher BMI ranges are at increased risk of cesarean delivery, even after accounting for the influence of parity, pregnancy complications, and hospital characteristics. ${ }^{15-18}$

Basic science studies examining mechanisms for slow labor progress suggest that in obese pregnant women, myometrial cells contract with reduced efficiency. ${ }^{19-21} \mathrm{~A}$ theoretical cause of myometrial inefficiency in settings of maternal obesity is lipotoxicity, whereby storage of excess free fatty acids in nonadipose tissues and use of fatty acids instead of glucose for cellular energy requirements causes widespread cellular damage and dysfunction. ${ }^{22-24}$ Highthroughput metabolomics, the comprehensive study of the end products and pathways of metabolism, offer a new capacity for the study of labor dystocia by allowing for the simultaneous measurement of trace biomarkers from several different mechanistic pathways. ${ }^{25-29}$ Thus, we conducted a case-control study to identify the metabolic profiles activated in the late second trimester/early third trimester serum of pregnant, African-American women who would go on to experience labor dystocia at term gestation. African-American women are nearly twice as likely as white women to be obese, $^{30}$ and 15 to $30 \%$ are more likely to have cesarean delivery. ${ }^{31,32}$ We hypothesized that African-American women with labor dystocia would have distinct metabolic profiles in late pregnancy compared with similar women who experienced normal active labor progression, and that metabolic changes consistent with lipotoxicity would predict labor dystocia in women with obesity.

\section{Methods}

\section{Study Design and Ethical Approvals}

Women from an ongoing prospective investigation (parent study) were included in this project. We obtained IRB approval from Emory University for this work. We conducted a matched case-control study, with a planned enrollment of 62 women ( 31 cases, 31 controls) based on an a priori power analysis. We used the MD Anderson Microarray Sample Size tool, appropriate for metabolomics data, to estimate power. Given that metabolomics data after log transformation are observed to follow an approximately normal distribution, 27 samples per group are required to reach $90 \%$ power (assuming a standard deviation of 0.7 , controlling for a fold change of 2 and false positive under 1 , which is consistent with prior analyses). ${ }^{33-37}$

\section{Study Participants}

To select cases and comparison controls for these analyses, all participants from the parent study ${ }^{38}$ (African-American women, age 18-45 years) were first assessed for eligibility. Women meeting inclusion criteria (term delivery [gestational age $\geq 37$ 0/7 weeks], nondiabetic (no gestational diabetes mellitus nor pre-existing diabetes], who completed active phase labor [ $4-10 \mathrm{~cm}$ of cervical dilation with regular contractions] with a nonanomalous singleton fetus in vertex presentation) had their medical records abstracted. Next, cases of labor dystocia and controls with normal labor progress were selected, matching individually on BMI at hospital admission $\left( \pm 3 \mathrm{~kg} / \mathrm{m}^{2}\right)$ and maternal age ( \pm 5 years).

Medical chart abstractions were conducted by two experienced clinicians using a standardized chart abstraction tool to ascertain maternal demographic factors, prenatal complications, gestational age at time of delivery, and details of delivery. Maternal delivery BMI was calculated from height at first prenatal visit and weight at the time of hospital admission for term labor (or at last prenatal visit occurring at 36 weeks gestation or later, if hospital admission weight was not available). Information on cervical progress was collected from each examination during labor. Repeat rater reliability testing was performed to confirm chart abstraction accuracy, showing greater than $98.0 \%$ accuracy on $10 \%$ of the abstracted charts. Clinical data was entered into REDCap and downloaded into R Statistical Software 3.5.2 (R Foundation Vienna, Austria) for data cleaning and statistical analyses.

Active phase labor durations were calculated for each woman who had regular, progressive contractions from 4 to $10 \mathrm{~cm}$ dilation. The slowest labors were chosen as cases, with controls selected from among the fastest labors while matching for maternal hospital admission BMI and age. Both BMI and age were also included as covariates in multivariate analyses to control for possible confounding caused these matching factors. $^{39}$ Investigators using metabolomics have shown that obesity causes profound changes in pregnant women that are distinguishable in a variety of body fluids and tissue samples and suggest multiple ways in which obesity changes metabolism. ${ }^{40,41}$ For this reason, we examined the withingroup influence of maternal delivery BMI by creating obese and nonobese groups (BMI at hospital admission $<$ or $\geq 30$ $\mathrm{kg} / \mathrm{m}^{2}$ ) of cases and controls for metabolomics analyses. Independent risk factors for labor dystocia were either used as exclusion criteria, balanced in case-control groups, used as covariates in multivariate analyses, or not controlled in analyses if they occurred during active phase labor as part of the causal pathway (-Supplementary Table 1). ${ }^{42,43}$

Frequencies and percentages were calculated for categorical variables describing maternal demographic, pregnancy, and labor characteristics across case-control groups; continuous data were assessed using median and interquartile range (non-normal distribution) or mean and standard deviation (normal distributions). Mann-Whitney $U$ test or $t$-tests were used for continuous data and likelihood ratio tests for categorical data. A 2-tailed $\alpha$ of 0.05 was used for determining statistical significance.

\section{High-Resolution Untargeted Metabolomics}

Serum samples (26-30 weeks gestation) from participants were transported on dry ice and maintained at $-80^{\circ} \mathrm{C}$ until 
analysis. Thawed samples were analyzed using high-resolution metabolomics platform, as described. ${ }^{35,37,44-46}$ Briefly, the samples were treated with acetonitrile $(2: 1, \mathrm{v} / \mathrm{v})$, spiked with internal standard mix, and centrifuged at $14,000 \times \mathrm{g}$ for 5 minutes at $4^{\circ} \mathrm{C}$ to remove proteins. Samples were maintained at $4^{\circ} \mathrm{C}$ in an autosampler until injection. A Thermo Scientific Q Exactive HF Quadruple-Orbitrap mass spectrometer (Thermo Fisher, San Diego, CA) was used, while an Orbitrap Fusion Tribrid mass spectrometer and an LTQ-Velos Orbitrap were used as backup as necessary. Dual chromatography (anion exchange and C18 reverse-phage columns) was used, and the coverage was enhanced by allowing positive and negative electrospray analysis of the same samples. Samples were analyzed in triplicate to improve reliable detection of low abundance metabolites. Samples were batch randomized, with case-control pairs randomized and analyzed within a single batch. For quality control purposes, six technical replicates were run at the beginning, middle, and end of each batch of 40 samples. Raw data files were extracted and aligned using apLCMS ${ }^{47}$ and xMSanalyzer. ${ }^{48}$ ComBat $^{49}$ was used prior to data analysis to correct for batch effects.

\section{Metabolomics Data Extraction}

The liquid chromatography mass-spectrometry (LC-MS/MS) data were processed by computational methods designed for high-resolution mass spectrometry ${ }^{47,48}$ to extract and quantify metabolite features. The resulting data contained 16,481 ions (defined by mass to charge ratio $[\mathrm{m} / \mathrm{z}]$ and retention time), which were filtered by detection quality metrics. For categorical variables, we checked for sparse cells and regrouped categories if necessary. For missing data, sensitivity analyses were performed to evaluate the impact of missing data on results, with consideration for implementing multiple imputation of missing values. All differential analyses were performed using xmsPANDA package v. 1.0.7.5. ${ }^{50}$ The comparison between groups with or without labor dystocia produced a statistic score to rank metabolic features, according to how likely they differentiated between the two groups. Features were filtered if they were present in less than $80 \%$ across all samples or were present in less than $80 \%$ in one group, $\log 2$ transformed, and quantile normalized. Missing features were replaced with median values. We selected different metabolite features from the comparison groups using linear regression with a $p$ value $<0.05$. Multiple hypothesis correction was performed using the Benjamini-Hochberg false discovery rate (FDR) correction method with threshold $q$-value of $0.20 .{ }^{51}$ Because the goal of this study was to explore metabolic differences among women with and without labor dystocia in pregnancy for future hypothesis-testing, we also used raw $p$-values for visualization methods and metabolic pathway enrichment analysis.

Analyses were also conducted after adjustment for maternal BMI and age. In building multivariable models, we checked linearity assumptions for continuous predictors and considered higher-order terms as appropriate. Type $1(-\log 10 \mathrm{p} \mathrm{vs} m / z)$ and Type $2(-\log 10 \mathrm{p}$ vs. retention time) Manhattan plots were created to visualize patterns of differentially expressed metabolites across all features with respect to molecular mass and chemical properties. Two-way hierarchical clustering analysis (HCA) was used to visualize relationship between women with and without labor dystocia in each group.

\section{Metabolic Pathway Analysis}

Metabolic pathways analysis was conducted with the topranked $(p<0.05)$ metabolite features using Mummichog 2.0 (http://mummichog.org/). ${ }^{44}$ Mummichog combines metabolite identification and metabolic pathway/network analysis. Significant metabolic patterns, even if not represented by conventional pathways, can be identified by Mummichog as clustered metabolic reactions. Pathway enrichment analysis thereby provides a two-step approach, which protects against both type I and type II errors. ${ }^{52}$ By this analysis, we identified distinct metabolic profiles/networks that were associated with labor dystocia at raw $p<0.05 .^{29,53-55}$ Metabolic pathway enrichment analyses were conducted on all samples with adjustment for maternal BMI and age, then again within obese and nonobese groups. Significant metabolic pathways with a minimum overlap size of 4 (e.g., number of metabolites with significant discrimination between cases and controls within a particular metabolic pathway) were evaluated for further analyses.

\section{Results}

A total of 287 medical record abstractions were completed. After excluding women who did not meet inclusion criteria or were missing key information on delivery BMI or cervical progress, a total of 165 labors remained. From these, 48 cases of labor dystocia and 49 normal labor progression controls were selected, matching on BMI at hospital admission and maternal age. Maternal demographic, pregnancy/labor characteristics, and labor outcome variables were similar between cases and controls $(p>0.05)$ within the obese and nonobese groups, with the exception of epidural anesthesia (higher in nonobese cases) (- Table 1). Median active phase labor duration $(4-10 \mathrm{~cm})$ was 10.9 hours in cases and 1.9 hours in controls across all maternal BMIs.

After quality filtering, a total of $8,792 / 16,481$ (53.3\%) of detected ions (defined by $\mathrm{m} / \mathrm{z}$ and retention time) were identified in mass spectral data from LC-MS/MS analysis of serum from participants for further analyses. Metabolic features that differentiated between labor dystocia cases and controls were selected in subgroups of obese women (140 features) and nonobese women (109 features) from the total sample. Using two-way HCA with these features, we observed separation between cases and controls that was also visible among significant features using principal components analysis (-Fig. 1, Ai-ii and Bi-ii). No individual metabolites were significant by FDR $q$-value of 0.20 .

Next, we examined metabolic pathways enriched in cases of labor dystocia within (a) the entire sample, (b) obese women, and (c) nonobese women ( - Fig. 1, A-iii and B-iii and - Table 2). Using the pathway enrichment program Mummichog, we determined which of the $m / z$ ions differing at raw $p<0.05$ were also enriched in specific metabolic pathways after adjusting for the influence of maternal BMI 
Labor Dystocia Metabolomics Carlson et al. e71

Table 1 Maternal demographic, pregnancy and labor characteristics, and labor outcomes by comparison groups

\begin{tabular}{|c|c|c|c|c|c|c|c|}
\hline & \multirow[t]{2}{*}{$\begin{array}{l}\text { Total sample } \\
(n=97)\end{array}$} & \multicolumn{2}{|c|}{ Labor dystocia case } & \multicolumn{2}{|c|}{ Normal labor progression control } & \multicolumn{2}{|c|}{$\begin{array}{l}p \text {-Value difference } \\
\text { between cases and } \\
\text { controls }\end{array}$} \\
\hline & & $\begin{array}{l}\mathrm{BMI}<30 \mathrm{~kg} / \mathrm{m}^{2} \\
(n=19)\end{array}$ & $\begin{array}{l}\mathrm{BMI} \geq 30 \mathrm{~kg} / \mathrm{m}^{2} \\
(n=29)\end{array}$ & $\begin{array}{l}\mathrm{BMI}<30 \mathrm{~kg} / \mathrm{m}^{2} \\
(n=20)\end{array}$ & $\begin{array}{l}\mathrm{BMI} \geq 30 \mathrm{~kg} / \mathrm{m}^{2} \\
(n=29)\end{array}$ & Nonobese $^{a}$ & Obese $^{\mathrm{b}}$ \\
\hline \multicolumn{8}{|l|}{ Maternal demographic } \\
\hline Race: African-American $(n, \%)$ & $97(100)$ & $19(100)$ & & $20(100)$ & & 1 & 1 \\
\hline Insurance status $(n, \%)$ & & & & & & 0.49 & 0.17 \\
\hline Private & 19 (19.6) & $2(10.5)$ & $8(27.6)$ & $4(20.0)$ & $5(17.2)$ & & \\
\hline Medicaid & $78(80.4)$ & $17(89.5)$ & $21(72.4)$ & $16(80.0)$ & $24(82.8)$ & & \\
\hline $\begin{array}{l}\text { Maternal age } \\
\text { (years, median } \pm I Q R \text { ) }\end{array}$ & $24.0(7)$ & $22.0(3)$ & $25.0(10)$ & $24.0(6)$ & $25.0(8)$ & 0.27 & 0.43 \\
\hline \multicolumn{8}{|l|}{ Pregnancy characteristics } \\
\hline $\begin{array}{l}\text { Prepregnancy BMI } \\
\left(\mathrm{kg} / \mathrm{m}^{2} \text {, median } \pm \mathrm{IQR}\right)\end{array}$ & $28.3(11.4)$ & $21.9(5.2)$ & $31.7(11.7)$ & $22.7(2.9)$ & $33.7(8.6)$ & 0.80 & 0.93 \\
\hline $\begin{array}{l}\text { Gestational weight gain } \\
(\mathrm{kg}, \text { median } \pm \mathrm{IQR})\end{array}$ & $11.3(8.8)$ & $10.4(7.7)$ & $14.0(14.0)$ & $10.5(6.3)$ & $9.5(9.6)$ & 0.71 & 0.70 \\
\hline $\begin{array}{l}\text { Delivery BMI } \\
\left(\mathrm{kg} / \mathrm{m}^{2}, \text { median } \pm \mathrm{IQR}\right)\end{array}$ & $32.6(10.3)$ & $26.9(4.7)$ & $36.7(10.1)$ & $26.7(2.6)$ & $37.0(7.5)$ & 0.95 & 0.35 \\
\hline Parity $(n, \%)$ & & & & & & 0.62 & 0.43 \\
\hline Nulliparous & $47(48.5)$ & $8(42.1)$ & $16(55.2)$ & $10(50.0)$ & $13(44.8)$ & & \\
\hline Multiparous & $50(51.5)$ & $11(57.9)$ & $13(44.8)$ & $10(50.0)$ & $16(55.2)$ & & \\
\hline \multicolumn{8}{|l|}{ Labor characteristics } \\
\hline $\begin{array}{l}\text { Gestational age at } \\
\text { labor admission } \\
\text { (weeks, days, median } \pm I Q R \text { ) }\end{array}$ & $39.2(1.9)$ & $38.6(2.0)$ & $39.4(1.4)$ & $38.6(1.6)$ & $39.3(2.0)$ & 0.67 & 0.59 \\
\hline Mode of labor onset $(n, \%)$ & & & & & & 0.64 & 1 \\
\hline Induced labor & $42(43.3)$ & $5(26.3)$ & $16(55.2)$ & $5(25.0)$ & $16(55.2)$ & & \\
\hline Spontaneous labor & $55(56.7)$ & $14(78.7)$ & $13(44.8)$ & $15(75.0)$ & $13(44.8)$ & & \\
\hline Cervical ripening in labor $(n, \%)$ & 29 (29.9) & $2(10.5)$ & $12(41.4)$ & $5(25.0)$ & $10(34.5)$ & 0.23 & 0.59 \\
\hline $\begin{array}{l}\text { Synthetic oxytocin in } \\
\text { labor }(n, \%)\end{array}$ & $59(60.8)$ & $11(57.9)$ & $23(82.1)$ & $9(45.0)$ & $16(55.2)$ & 0.42 & 0.03 \\
\hline $\begin{array}{l}\text { Type of membrane } \\
\text { rupture }(n, \%)^{c}\end{array}$ & & & & & & 0.27 & 0.16 \\
\hline Spontaneous & $47(48.5)$ & $9(47.4)$ & $9(31.0)$ & $14(70.0)$ & $15(51.7)$ & & \\
\hline Artificial & $49(50.5)$ & $10(52.6)$ & $19(65.5)$ & $6(30.0)$ & $14(48.3)$ & & \\
\hline Epidural in labor $(n, \%)$ & $73(75.3)$ & $16(84.2)$ & $25(86.2)$ & $11(55.0)$ & $21(72.4)$ & 0.04 & 0.19 \\
\hline $\begin{array}{l}\text { Active labor duration } \\
\text { (hours, median } \pm I Q R \text { ) }\end{array}$ & $6.5(8.9)$ & $8.25(6.8)$ & $11.4(4.9)$ & $1.35(2.0)$ & $2.5(2.3)$ & $<0.001$ & $<0.001$ \\
\hline \multicolumn{8}{|l|}{ Labor outcomes } \\
\hline Type of delivery $(n, \%)$ & & & & & & 0.08 & 0.09 \\
\hline Spontaneous vaginal & $84(86.6)$ & $17(89.5)$ & $23(79.3)$ & $20(100)$ & $24(82.8)$ & & \\
\hline Vacuum-assisted vaginal & $11(11.3)$ & $2(10.5)$ & $4(13.8)$ & 0 & $5(17.2)$ & & \\
\hline Cesarean & $2(2.1)$ & 0 & $2(6.9)$ & 0 & 0 & & \\
\hline $\begin{array}{l}\text { Postpartum hemorrhage } \\
(>500 \mathrm{~mL}, n \%)\end{array}$ & $6(6.2)$ & 0 & $4(13.8)$ & 0 & $2(6.9)$ & - & 0.38 \\
\hline Newborn gender $(n, \%)$ & & & & & & 0.87 & 1 \\
\hline Male & $50(51.5)$ & $10(52.6)$ & $15(51.7)$ & $10(50)$ & $15(51.7)$ & & \\
\hline Female & $47(48.5)$ & $9(47.4)$ & $14(48.3)$ & $10(50)$ & $14(48.3)$ & & \\
\hline Newborn NICU admission $(n, \%)$ & $5(5.3)$ & 0 & $2(6.9)$ & $2(10.5)$ & $1(3.6)$ & 0.09 & 0.57 \\
\hline $\begin{array}{l}\text { Neonatal birthweight } \\
\text { (grams, mean } \pm \text { SD) }\end{array}$ & $\begin{array}{l}3119.3 \\
(394.4)\end{array}$ & $2965(571.0)$ & $3060.0(533.0)$ & $3070.0(446.5)$ & $3175.0(465.0)$ & 0.83 & 0.42 \\
\hline
\end{tabular}

Abbreviations: BMI, body mass index; IQR, interquartile range; NICU, neonatal intensive care unit; SD, standard deviation.

a,b $p$-Value for comparison of cases and controls within nonobese $e^{a}$ and obese ${ }^{b}$ groups. Likelihood ratio significance testing for categorical variables and Mann-Whitney $\mathrm{U}$ test used for continuous variables. $p$-Values $<0.05$ are shown in bold font.

"One case of "uncertain" method for rupture of membranes in labor dystocia case in obese group. 


\section{Obese Women}

i.

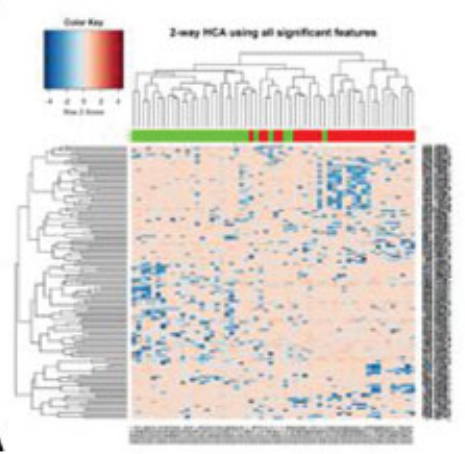

A ii.

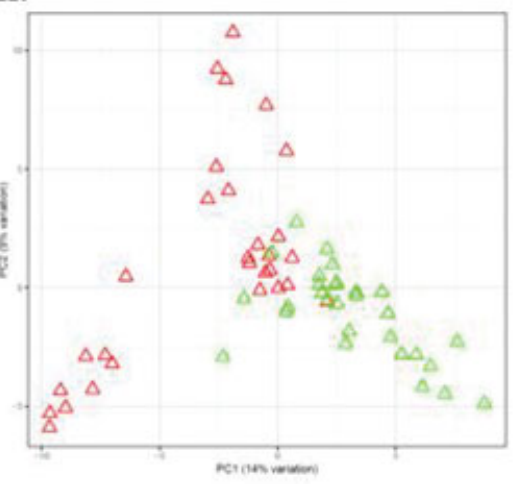

iii.

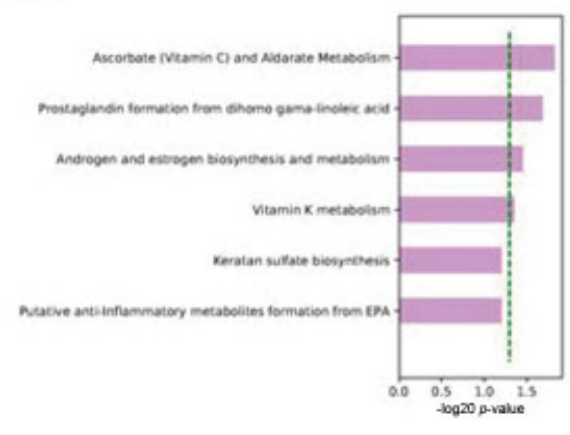

\section{Non-Obese Women}

i.

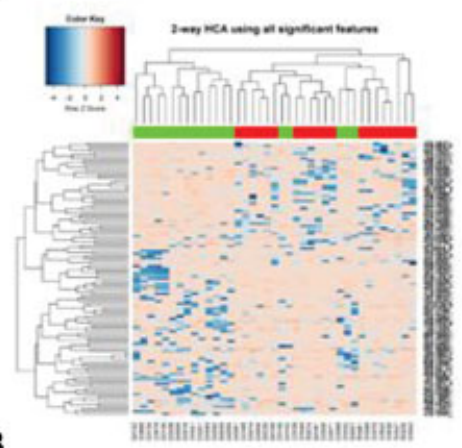

ii.

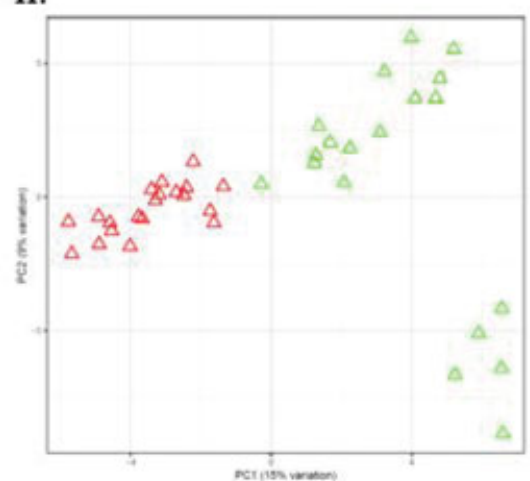

iii.

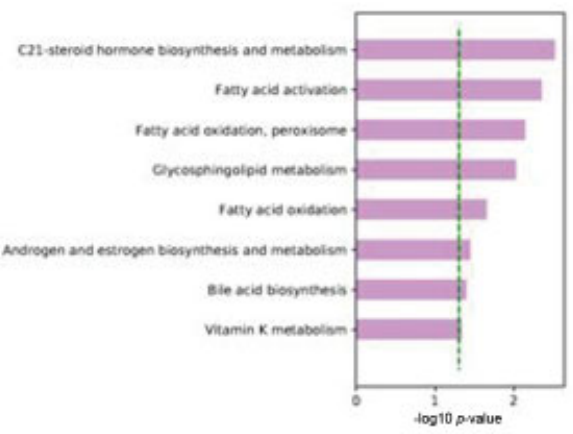

Fig. 1 Two-way hierarchical cluster analysis (HCA) (i), principal components analysis (PCA) (ii), and metabolic pathway analysis (iii) showing metabolites and metabolic pathways differentially expressed between women with labor dystocia and normal labor controls among (A) obese and (B) nonobese women caption: Each column in HCA represents a participant and each row represents a metabolite feature. Red hues indicate metabolites with enhanced levels, and blue hues represent metabolites with lower metabolite concentrations. Labor dystocia is represented in green and normal labor in red across $x$-axis. Red triangles in PCA represent normal labor and green triangles represent labor dystocia. (A) Obese women, (i) HCA with top 140/8813 metabolites with a raw $p<0.05$ is shown and (ii) PCA and (iii) Mummichog enriched metabolic pathways differentiating labor dystocia in matched groups (B) Nonobese women, (i) HCA with top 109/8704 metabolites with a raw $p<0.05$ is shown and (ii) PCA and (iii) Mummichog enriched metabolic pathways differentiating labor dystocia in matched groups of (A) obese and (B) nonobese women shown with dotted green line represents pathways significant at $p<0.05$. Metabolic pathway analyses include adjustment within each group for maternal body mass index (continuous, at hospital admission for labor) and maternal age.

Table 2 Metabolite pathways significantly dysregulated in labor dystocia in multivariate analyses

\begin{tabular}{|c|c|c|c|c|c|c|c|c|c|}
\hline \multirow[t]{2}{*}{ Pathways } & \multicolumn{3}{|c|}{ Total sample } & \multicolumn{3}{|c|}{$\mathrm{BMI}<30 \mathrm{~kg} / \mathrm{m}^{2}$} & \multicolumn{3}{|c|}{$\mathrm{BMI} \geq 30 \mathrm{~kg} / \mathrm{m}^{2}$} \\
\hline & $\begin{array}{l}\text { Overlap } \\
\text { size }\end{array}$ & $\begin{array}{l}\text { Pathway } \\
\text { size }\end{array}$ & $p$-Value & $\begin{array}{l}\text { Overlap } \\
\text { Size }\end{array}$ & $\begin{array}{l}\text { Pathway } \\
\text { size }\end{array}$ & $p$-Value & $\begin{array}{l}\text { Overlap } \\
\text { Size }\end{array}$ & $\begin{array}{l}\text { Pathways } \\
\text { size }\end{array}$ & $p$-Value \\
\hline $\begin{array}{l}\text { C21-steroid hormone } \\
\text { biosynthesis and metabolism }\end{array}$ & 11 & 64 & 0.001 & 9 & 60 & 0.003 & & & \\
\hline Glycosphingolipid metabolism & 5 & 30 & 0.017 & 5 & 30 & 0.010 & & & \\
\hline Tryptophan metabolism & 7 & 53 & 0.021 & & & & & & \\
\hline Fatty acid activation & & & & 6 & 33 & 0.005 & & & \\
\hline $\begin{array}{l}\text { Androgen and estrogen } \\
\text { biosynthesis and metabolism }\end{array}$ & 6 & 45 & 0.028 & 5 & 42 & 0.036 & 5 & 45 & 0.035 \\
\hline Bile acid biosynthesis & & & & 4 & 31 & 0.041 & & & \\
\hline
\end{tabular}

Abbreviations: BMI, body mass index; C21, 21 carbon.

Significant metabolite pathways predicting labor dystocia shown for total sample and in subgroups defined by maternal obesity using raw $p$-value multivariate analysis with adjustment within each group for maternal BMI (continuous, at hospital admission for labor) and maternal age. Pathways with an overlap size of at least 4 are shown (i.e., pathway included at least 4 metabolites that significantly differentiated the case-control groups). 
and age. Across all three comparison groups, cases of labor dystocia differed from normal labor controls in androgen and estrogen biosynthesis pathway metabolites (total sample, $p=0.028$, overlap size 6 of 45 ; nonobese women, $p=0.036$, overlap size 5 of 42 ; obese women, $p=0.035$, overlap size 5 of 45).

Among women in the nonobese group, C21-steroid hormone biosynthesis and metabolism pathway metabolites significantly differentiated labor dystocia cases and controls. This metabolic pathway also differentiated women with labor dystocia in the total sample after adjusting for the influence of maternal BMI and age, but did not differentiate women in the obese group who experienced labor dystocia. As measured in the untargeted LC-MS/MS, serum levels of metabolites in the C21-steroid pathway were lower among women who went on to experience labor dystocia compared with control samples.

Similar to the C21-steroid pathway findings, glycosphingolipid pathway metabolites were also overrepresented among the features discriminating women with labor dystocia from controls in both the total sample and nonobese group, but not the obese group (-Table 2 ). By contrast, only women in the nonobese group with labor dystocia were differentiated from normal labor controls by metabolites mapped to the bile acid biosynthesis and fatty acid activation pathways. Among $\mathrm{m} / \mathrm{z}$ features linked to the bile acid biosynthesis and fatty acid activation pathways, all were relatively higher in serum from women with normal labor progression, compared with nonobese women who experienced labor dystocia (0.23-3.59-fold increase in controls vs. cases, $p=0.04-0.47)$. Neither of these pathways differentiated labor dystocia case-controls in the obese group nor in the total sample once maternal BMI and age were included in multivariate models.

When the influence of maternal BMI and age was held constant, tryptophan metabolism significantly differentiated labor dystocia cases and controls across all women ( - Table 2 ). Among metabolites tentatively matched from this pathway to untargeted LC-MS/MS ions, women with normal labor progression had relatively higher circulating serum levels than women with labor dystocia (1.32-2.28-fold increase in controls vs. cases, $p=0.03-0.004)$.

\section{Discussion}

In this case-control study, we present evidence that labor dystocia was predicted by different metabolic pathways in late second/early third trimester serum among obese versus nonobese African-American women, and only androgen and estrogen biosynthesis pathway metabolites predicted labor dystocia across both obese and nonobese groups. A range of factors can slow labor progress, thereby increasing women's risk for unplanned cesarean birth. However, there is growing recognition that in some women, there is a phenotype of labor dystocia that complicates the labor process and can worsen labor outcomes. ${ }^{17,24}$ Maternal BMI above normal ranges appears to be one part of this labor dystocia phenotype, as evidenced by clinical investigations showing increases in labor length $^{13,56}$ and risk for cesarean delivery ${ }^{57}$ among women with higher BMIs. However, labor dystocia is not confined to obese women. ${ }^{21,38,39}$ Additionally, not all pregnant women with obesity go on to experience labor dystocia and cesarean birth. ${ }^{58}$ Thus, there is a need to identify biomarkers of labor dystocia. $^{12}$

Sex hormone metabolism was associated in this study with labor dystocia in both the obese and nonobese groups, and in the total sample after adjustment for maternal age and BMI. We repeated metabolomics analyses while adjusting for fetal gender, but saw no difference in associations between labor dystocia and sex hormone metabolism pathways (analyses not shown). In other investigations, women with obesity were noted to have increased levels of estrogen and testosterone both within ${ }^{59}$ and outside of pregnancy. ${ }^{60}$ In addition, lower serum levels of estrogen and progesterone ${ }^{61}$ and single nucleotide polymorphisms of sex hormone-related genes ${ }^{62}$ were associated with preeclampsia in two recent investigations. We did not locate published studies reporting links between sex hormone metabolites and labor dystocia. However, given close links between maternal obesity and both hypercholesterolemia ${ }^{63}$ and preeclampsia, ${ }^{64}$ further research with targeted measures of specific sex and steroid hormones in women with active labor slowing is warranted.

Contrary to our prediction that metabolic changes consistent with lipotoxicity would predict labor dystocia in women with obesity, we found more evidence for associations between lipotoxicity and labor dystocia among nonobese women in our sample. Both fatty acid activation and glycosphingolipid metabolism pathways were increased in nonobese women who had fast active labor progression (controls). These observations hint that more efficient mobilization of lipids from the diet and adipose tissue for use by energy-requiring tissues may protect pregnant women from cellular damage and dysregulation associated with lipotoxicity, thereby leading to faster labor progression. We also observed decreases in C21-steroid hormone metabolites among nonobese women who went on to experience labor dystocia. The $\mathrm{C} 21$ steroid hormone metabolic pathway involves production of both progestins and adrenal corticosteroids, and is closely related to the C-19 and C-18 pathways, which produce androgens and estrogens, respectively. ${ }^{65}$

There is evidence from mouse and human models that increased fatty acid oxidation decreases systemic free fatty acid and dyslipidemia ${ }^{66,67}$-both landmarks of lipotoxicity. In addition, glycosphingolipid metabolism-the other pathway associated with labor dystocia among nonobese women in this study-is also related to lipotoxicity. Glycosphingolipids are a group of bioactive glycolipids that are involved in signal transduction, cholesterol lipid rafts, and cellular communication. ${ }^{68}$ Our observation of relatively lower levels of metabolites tentatively mapped to glycosphingolipid metabolism in nonobese women with labor dystocia is interesting, given evidence that cholesterol lipid rafts may be involved with an unstable, asynchronous uterine contractile pattern in a mouse model. ${ }^{69}$ Myometrial lipotoxicity involving accumulation of lipids and reduction in muscle mass in uterine tissue from obese pregnant women were recently demonstrated by Gam et al. ${ }^{22}$ Building on these findings, we theorize that labor dystocia 
cases among nonobese women in our investigation were more likely than normal-labor comparison subjects to have lipotoxicity-related changes in their uterus that ultimately slowed their labor progression. For example, as a result of problems with fatty acid oxidation, their myometrial tissue may have accumulated lipid deposits, thereby reducing contractile force and frequency during labor and slowing cervical dilation. In addition, glycosphingolipid metabolite alterations may have decreased these women's capacity to maintain a stable, synchronous uterine contractile pattern-thereby further impeding labor progress.

As mentioned above, we did not find evidence for associations between lipotoxicity and labor dystocia among obese women in our sample. It is possible that lipotoxicity may be a more important differentiator of the labor dystocia phenotype among women who are not yet obese, but show evidence of less effective metabolism of fats. It is not known how circulating levels of metabolites from the glycosphingolipid or fatty acid activation pathways in obese women compared with nonobese women in our sample, as no $m / z$ features from these pathways were also annotated at a high confidence level. However, lower fatty acid oxidation and lipid accumulation have been correlated with higher maternal BMIs in umbilical stem cells of neonates born to obese versus normal weight women. ${ }^{70}$ These data suggest that reduced lipid oxidation during pregnancy is an important facet of obesity-related changes in the maternal-fetal pair. It is possible that perturbed fatty acid activation and glycosphingolipid pathways were therefore present in most or all of the women from our sample of obese women, thereby reducing heterogeneity of these pathways for the purposes of our metabolic pathway analysis. Future targeted metabolomics investigations are needed to quantify levels of metabolites involved in lipotoxicity among women of different BMIs and compare lipotoxicity metabolite exposure to labor durations.

Also supported in the literature was our unexpected finding of relatively higher levels of features linked to the tryptophan metabolism pathway in women with normal labor progress after adjustment for the influence of maternal BMI and age. The tryptophan metabolic pathway is important during pregnancy for fetal development, neuronal protection, and serotonin signaling. ${ }^{71}$ Serotonin signaling deficiencies may be linked to depression or anxiety in pregnancy, and both maternal trauma history ${ }^{72}$ and prenatal depression and anxiety ${ }^{73-75}$ were associated with labor dysfunction in previous clinical investigations. In addition, serotonin stimulates uterine smooth muscle contractions in pregnant women. ${ }^{76}$ Thus, relative upregulation of tryptophan metabolism in women with normal labor progress could suggest possible links between maternal mood, uterine contractility, and labor duration. However, further investigation is needed to target metabolites of the tryptophan metabolic pathway, including serotonin, in pregnant women who experience labor dystocia and other difficulties of parturition.

The application of metabolomics to understand pregnancy risks is still a young field. In 2009, the first review on this topic was published based on limited data. ${ }^{77}$ Since then, emerging studies demonstrate the promising possibilities of this technology in the complex field of pregnancy where a mother- baby pair are interacting for mutual benefit. ${ }^{78-82}$ Most recently, metabolomics was used to identify specific metabolites that were predictive of women's eventual development of preeclampsia ${ }^{83,84}$ and of their neonate's level of adiposity. ${ }^{85}$

Metabolomics is also useful for understanding labor processes. Several teams of investigators differentiated between women in different phases of labor using metabolomics. ${ }^{86,87}$ As mentioned, metabolic profiles can be generated from a variety of biological fluids; this methodologic advantage was recently utilized to demonstrate biomarkers present in amniotic fluid and maternal serum collected during women's second trimester that predicted later preterm delivery. ${ }^{88}$ Metabolomics has also been used to identify biomarkers produced in mice uterine tissue following pharmacologic stimulation versus inhibition of contractions, revealing new detail on the mechanisms of uterine contractility in rodents. ${ }^{89}$

Our study has several strengths. First, potential differences in labor duration that could potentially be caused by differences in labor management were offset in this study by our focus on participants who were managed by the same team of physicians and midwives following the same protocols across two hospitals. ${ }^{42}$ Another strength of this study is our careful consideration of variables known to associate with the duration of labor in our choice of inclusion/exclusion criteria, case-control group assignment, and statistical covariates. A range of factors can affect labor progress, including maternal age, ${ }^{90} \mathrm{BMI},{ }^{13}$ maternal parity, ${ }^{11}$ and mode of labor onset. ${ }^{91}$ In this study, we adjusted for the influence of maternal age, and matched cases with controls on mode of labor onset and BMI. In bivariate comparisons, we saw no difference after controlling for the abovementioned factors in maternal parity. An additional strength was our use of high-resolution LC-MS/MS combined with powerful analytic tools to provide broad coverage of both low- and high-abundance metabolites that are potentially relevant to labor dystocia pathophysiology.

Our study also has several limitations. First, the retrospective nature of this analysis means that we cannot differentiate between cause and effect, but only on associations between metabolic pathways present in late pregnancy serum and term labor dystocia. Second, although our sample size exceeded a priori calculations, larger numbers of participants-especially in the nonobese group-would strengthen these analyses. In addition, our metabolomics samples were collected in the late second/early third trimester of pregnancy. Future investigations might better capture the metabolic signature of a labor dystocia phenotype by also including a first trimester sample and a late third trimester sample. With these repeated measures, a longitudinal analysis might be conducted where intraindividual might be filtered out, leaving a clearer signal for significant $m / z$ features and metabolic pathways distinguishing women with labor progression difficulty. We were also not able to consider whether the timing of late-pregnancy serum influenced the strength of metabolic signals in this analysis due to small sample size. Future longitudinal analysis with larger groups of samples would allow us to consider how metabolic levels in these critical pathways might vary across pregnancy. In our analyses of individual metabolites, we were unable to correct for multiple comparisons, as no metabolite 
reached the FDR $q$-value $<0.20$. Possibly, in a future analysis involving longitudinal measures or standardization in sample collection by time of day and dietary intake, this threshold would be reached. However, our use of metabolic pathway analysis using features significant at raw $p$-value provides a 2 step approach that protects against both type I and type II errors while revealing networks of perturbed metabolites. ${ }^{52}$ This approach allowed us to explore metabolic differences among women with and without labor dystocia in pregnancy for future hypothesis-testing using targeted metabolomics approaches while employing orthogonal methods to confirm findings. Finally, our study is limited by our inclusion of only one race of women for all analyses. However, given the disparities experienced by African-American women in rates of cesarean delivery ${ }^{92}$ and maternal morbidity/mortality ${ }^{93,94}$ and the benefits of looking intrarace for differences in highthroughput methodologies like metabolomics, ${ }^{95}$ this study's focus on one race is a logical choice. Metabolic pathways associated with labor dystocia in African-American women from this study should be further investigated in future studies that might include samples with a range of maternal races, standardization in sample collection by time of day and dietary intake, and targeted analysis of associated metabolites.

We need new approaches to improve birth outcomes in women with a labor dystocia phenotype. ${ }^{58,96,97}$ As a methodology that covers the spectrum from hypothesis development to hypothesis testing, metabolomics offers investigators the ability to quantify multiple metabolites in a single sample and flexibility in the type and amount of sample required for analyses. Thus, metabolomics offers considerable promise in the investigation of mechanisms contributing to labor dystocia among women of different race/ethnicities, with or without obesity. With a fuller understanding of the unique metabolic mechanisms contributing to labor dystocia, we step closer to breaking the cascade of health complications resulting from poor labor outcomes.

Declaration of Conflicting Interests per International Committee of Medical Journal Editors Guidelines

The authors declare that there is no conflict of interest.

Funding

Drs. Nicole Carlson and Jennifer Frediani were supported by grant number K01NR016984 from the National Institute of Nursing Research during manuscript production. This manuscript was supported by funding from the National Institutes of Health National Institutes of Nursing Research (R01NR014800), the Office of the Director [3R01NR014800, UG3OD023318], the National Institute of Environmental Health Sciences (P50ES926071), and the US Environmental Protection Agency (83615301 Mod. 2).

\section{Acknowledgments}

The authors would like to thank Sudeshna Paul, PhD, for her assistance with statistical interpretation and format of tables and Rebecca M. Mitchell, PhD, for her assistance with $\mathrm{R}$ programming for data cleaning and analysis.

\section{References}

1 Hamilton BE, Martin JA, Osterman MJ. Births: preliminary data for 2015. Natl Vital Stat Rep 2016;65(03):1-15

2 The Joint Commission. Specifications Manual for Joint Commission National Quality Measures. Joint Commission 2016. Available at: https://manual.jointcommission.org/releases/TJC2016A/MIF0167. html

3 Osterman MJ, Martin JA. Trends in low-risk cesarean delivery in the United States, 1990-2013. Natl Vital Stat Rep 2014;63(06):1-16

4 Martin JA, Hamilton BE, Osterman MJ, Driscoll AK, Drake P. Births: Final data for 2017. Hyattsville, MD: National Center for Health Statistics; 2018

5 Clark EA, Silver RM. Long-term maternal morbidity associated with repeat cesarean delivery. Am J Obstet Gynecol 2011;205(6, Suppl):S2-S10

6 Silver RM, Landon MB, Rouse DJ, et al; National Institute of Child Health and Human Development Maternal-Fetal Medicine Units Network. Maternal morbidity associated with multiple repeat cesarean deliveries. Obstet Gynecol 2006;107(06):1226-1232

7 Liu S, Liston RM, Joseph KS, Heaman M, Sauve R, Kramer MS; Maternal Health Study Group of the Canadian Perinatal Surveillance System. Maternal mortality and severe morbidity associated with low-risk planned cesarean delivery versus planned vaginal delivery at term. CMAJ 2007;176(04):455-460

8 Petersen EE, Davis NL, Goodman D, et al. Vital signs: pregnancyrelated deaths, United States, 2011-2015, and strategies for prevention, 13 states, 2013-2017. MMWR Morb Mortal Wkly Rep 2019;68(18):423-429

9 Spong CY, Berghella V, Wenstrom KD, Mercer BM, Saade GR. Preventing the first cesarean delivery: summary of a joint Eunice Kennedy Shriver National Institute of Child Health and Human Development, Society for Maternal-Fetal Medicine, and American College of Obstetricians and Gynecologists Workshop. Obstet Gynecol 2012;120(05):1181-1193

10 Barber EL, Lundsberg LS, Belanger K, Pettker CM, Funai EF, Illuzzi $\mathrm{JL}$. Indications contributing to the increasing cesarean delivery rate. Obstet Gynecol 2011;118(01):29-38

11 Zhang J, Landy HJ, Branch DW, et al; Consortium on Safe Labor. Contemporary patterns of spontaneous labor with normal neonatal outcomes. Obstet Gynecol 2010;116(06):1281-1287

12 Neal JL, Lowe NK, Patrick TE, Cabbage LA, Corwin EJ. What is the slowest-yet-normal cervical dilation rate among nulliparous women with spontaneous labor onset? J Obstet Gynecol Neonatal Nurs 2010;39(04):361-369

13 Kominiarek MA, Zhang J, Vanveldhuisen P, Troendle J, Beaver J, Hibbard JU. Contemporary labor patterns: the impact of maternal body mass index. Am J Obstet Gynecol 2011;205(03):244.e1-244.e8

14 Cedergren MI. Non-elective caesarean delivery due to ineffective uterine contractility or due to obstructed labour in relation to maternal body mass index. Eur J Obstet Gynecol Reprod Biol 2009; 145(02):163-166

15 Chu SY, Kim SY, Schmid CH, et al. Maternal obesity and risk of cesarean delivery: a meta-analysis. Obes Rev 2007;8(05):385-394

16 Vahratian A, Siega-Riz AM, Savitz DA, Zhang J. Maternal prepregnancy overweight and obesity and the risk of cesarean delivery in nulliparous women. Ann Epidemiol 2005;15(07):467-474

17 Hautakangas T, Palomäki O, Eidstø K, Huhtala H, Uotila J. Impact of obesity and other risk factors on labor dystocia in term primiparous women: a case control study. BMC Pregnancy Childbirth 2018;18(01):304

18 Tse WT, Chaemsaithong P, Chan WWY, et al. Labor progress determined by ultrasound is different in women requiring cesarean delivery from those who experience a vaginal delivery following induction of labor. Am J Obstet Gynecol 2019;221 (04):335.e1-335.e18

19 Parkington HC, Stevenson J, Tonta MA, et al. Diminished hERG K+ channel activity facilitates strong human labour contractions but is dysregulated in obese women. Nat Commun 2014;5:4108 
20 Shmygol A, Noble K, Wray S. Depletion of membrane cholesterol eliminates the $\mathrm{Ca} 2+$-activated component of outward potassium current and decreases membrane capacitance in rat uterine myocytes. J Physiol 2007;581(Pt 2):445-456

21 Jie Zhang, Kendrick A, Quenby S, Wray S. Contractility and calcium signaling of human myometrium are profoundly affected by cholesterol manipulation: implications for labor? Reprod Sci 2007; 14(05):456-466

22 Gam CMBF, Larsen LH, Mortensen OH, et al. Unchanged mitochondrial phenotype, but accumulation of lipids in the myometrium in obese pregnant women. J Physiol 2017;595(23): 7109-7122

23 Frayn KN. Metabolic Regulation: A Human Perspective. 3rd edition. Chichester, U.K.; Malden, MA: Wiley-Blackwell Pub.; 2010

24 Carlson NS, Hernandez TL, Hurt KJ. Parturition dysfunction in obesity: time to target the pathobiology. Reprod Biol Endocrinol 2015;13(01):135

25 Jones DP. Redefining oxidative stress. Antioxid Redox Signal 2006; 8(9-10):1865-1879

26 Abu Bakar MH, Sarmidi MR, Cheng KK, et al. Metabolomics - the complementary field in systems biology: a review on obesity and type 2 diabetes. Mol Biosyst 2015;11(07):1742-1774

27 Jones DP, Park Y, Ziegler TR. Nutritional metabolomics: progress in addressing complexity in diet and health. Annu Rev Nutr 2012; 32:183-202

28 Patti GJ, Yanes O, Siuzdak G. Innovation: Metabolomics: the apogee of the omics trilogy. Nat Rev Mol Cell Biol 2012;13(04): 263-269

29 Xie B, Waters MJ, Schirra HJ. Investigating potential mechanisms of obesity by metabolomics. J Biomed Biotechnol 2012;2012:805683

30 Ogden CL, Carroll MD, Kit BK, Flegal KM. Prevalence of childhood and adult obesity in the United States, 2011-2012. JAMA 2014; 311(08):806-814

31 Martin JA, Hamilton BE, Osterman MJK, Curtin SC, Matthews TJ. Births: Final Data for 2013. Hyattsville, MD: National Center for Health Statistics; 2015

32 Aron DC, Gordon HS, DiGiuseppe DL, Harper DL, Rosenthal GE. Variations in risk-adjusted cesarean delivery rates according to race and health insurance. Med Care 2000;38(01):35-44

33 Cribbs SK, Park Y, Guidot DM, et al. Metabolomics of bronchoalveolar lavage differentiate healthy HIV-1-infected subjects from controls. AIDS Res Hum Retroviruses 2014;30(06):579-585

34 Fitzpatrick AM, Park Y, Brown LA, Jones DP. Children with severe asthma have unique oxidative stress-associated metabolomic profiles. J Allergy Clin Immunol 2014;133(01):258-61.e1, 8

35 Roede JR, Uppal K, Park Y, et al. Serum metabolomics of slow vs. rapid motor progression Parkinson's disease: a pilot study. PLoS One 2013;8(10):e77629

36 Frediani JK, Jones DP, Tukvadze N, et al. Plasma metabolomics in human pulmonary tuberculosis disease: a pilot study. PLoS One 2014;9(10):e108854

37 Neujahr DC, Uppal K, Force SD, et al. Bile acid aspiration associated with lung chemical profile linked to other biomarkers of injury after lung transplantation. Am J Transplant 2014;14(04):841-848

38 Corwin EJ, Hogue CJ, Pearce B, et al. Protocol for the Emory University African American Vaginal, Oral, and Gut Microbiome in Pregnancy Cohort Study. BMC Pregnancy Childbirth 2017;17 (01):161

39 Pearce N. Analysis of matched case-control studies. BMJ 2016; 352:1969

40 Hellmuth C, Lindsay KL, Uhl O, et al. Association of maternal prepregnancy BMI with metabolomic profile across gestation. Int J Obes 2017;41(01):159-169

41 Fattuoni C, Mandò C, Palmas F, et al. Preliminary metabolomics analysis of placenta in maternal obesity. Placenta 2018;61:89-95

42 Rogers AJG, Harper LM, Mari G. A conceptual framework for the impact of obesity on risk of cesarean delivery. Am J Obstet Gynecol 2018;219(04):356-363
43 Snowden JM, Tilden EL, Odden MC. Formulating and answering high-impact causal questions in physiologic childbirth science: concepts and assumptions. J Midwifery Womens Health 2018;63 (06):721-730

44 Li S, Park Y, Duraisingham S, et al. Predicting network activity from high throughput metabolomics. PLOS Comput Biol 2013;9 (07):e1003123

45 Soltow QA, Zeanah EH, Lira VA, Criswell DS. Cessation of cyclic stretch induces atrophy of C2C12 myotubes. Biochem Biophys Res Commun 2013;434(02):316-321

46 Hoffman JM, Soltow QA, Li S, Sidik A, Jones DP, Promislow DE. Effects of age, sex, and genotype on high-sensitivity metabolomic profiles in the fruit fly, Drosophila melanogaster. Aging Cell 2014; 13(04):596-604

$47 \mathrm{Yu}$ T, Park Y, Li S, Jones DP. Hybrid feature detection and information accumulation using high-resolution LC-MS metabolomics data. J Proteome Res 2013;12(03):1419-1427

48 Uppal K, Soltow QA, Strobel FH, et al. xMSanalyzer: automated pipeline for improved feature detection and downstream analysis of large-scale, non-targeted metabolomics data. BMC Bioinformatics 2013;14:15

49 Johnson WE, Li C, Rabinovic A. Adjusting batch effects in microarray expression data using empirical Bayes methods. Biostatistics 2007;8(01):118-127

50 Smyth GK. Linear models and empirical bayes methods for assessing differential expression in microarray experiments. Stat Appl Genet Mol Biol 2004; doi: 10.2202/1544-6115.1027

51 Hochberg Y, Benjamini Y. More powerful procedures for multiple significance testing. Stat Med 1990;9(07):811-818

52 Uppal K, Walker DI, Liu K, Li S, Go YM, Jones DP. Computational metabolomics: a framework for the Million Metabolome. Chem Res Toxicol 2016;29(12):1956-1975

53 Serkova NJ, Jackman M, Brown JL, et al. Metabolic profiling of livers and blood from obese Zucker rats. J Hepatol 2006;44(05): 956-962

54 Desert R, Canlet C, Costet N, Cordier S, Bonvallot N. Impact of maternal obesity on the metabolic profiles of pregnant women and their offspring at birth. Metabolomics 2015;11:1896-1907

55 Zhou Y, Qiu L, Xiao Q et al. Obesity and diabetes related plasma amino acid alterations. Clin Biochem 2013;46(15):1447-1452

56 Norman SM, Tuuli MG, Odibo AO, Caughey AB, Roehl KA, Cahill AG. The effects of obesity on the first stage of labor. Obstet Gynecol 2012;120(01):130-135

57 Kominiarek MA, Vanveldhuisen P, Hibbard J, et al; Consortium on Safe Labor. The maternal body mass index: a strong association with delivery route. Am J Obstet Gynecol 2010;203(03):264.e1-264.e7

58 Pevzner L, Powers BL, Rayburn WF, Rumney P, Wing DA. Effects of maternal obesity on duration and outcomes of prostaglandin cervical ripening and labor induction. Obstet Gynecol 2009;114 (06):1315-1321

59 Berkane N, Liere P, Oudinet JP, et al. From pregnancy to preeclampsia: a key role for estrogens. Endocr Rev 2017;38(02): 123-144

60 Palmer BF, Clegg DJ. The sexual dimorphism of obesity. Mol Cell Endocrinol 2015;402:113-119

61 Wan J, Hu Z, Zeng K, et al. The reduction in circulating levels of estrogen and progesterone in women with preeclampsia. Pregnancy Hypertens 2018;11:18-25

62 Zhang P, Yang H, Feng Y, et al. Polymorphisms in sex hormone metabolism genes and risk of preeclampsia in Taiyuan, China. Gynecol Obstet Invest 2018;83(02):179-186

63 Gostynski M, Gutzwiller F, Kuulasmaa K, et al; WHO MONICA Project. Analysis of the relationship between total cholesterol, age, body mass index among males and females in the WHO MONICA Project. Int J Obes Relat Metab Disord 2004;28(08):1082-1090

64 Bodnar LM, Catov JM, Klebanoff MA, Ness RB, Roberts JM. Prepregnancy body mass index and the occurrence of severe hypertensive disorders of pregnancy. Epidemiology 2007;18(02):234-239 
65 Mesiano S. Endocrinology of human pregnancy and fetal-placental neuroendocrine development. In: Strauss J, Barbieri R, Gargiulo A, eds. Yen \& Jaffe's Reproductive Endocrinology. 8th edition. Elsevier; 2019:1001

66 Zhang S, Chen G, Li N, et al. CYP2J2 overexpression ameliorates hyperlipidemia via increased fatty acid oxidation mediated by the AMPK pathway. Obesity 2015;23(07):1401-1413

67 Badoud F, Perreault M, Zulyniak MA, Mutch DM. Molecular insights into the role of white adipose tissue in metabolically unhealthy normal weight and metabolically healthy obese individuals. FASEB J 2015;29(03):748-758

$68 \mathrm{Xu} \mathrm{YH,} \mathrm{Barnes} \mathrm{S,} \mathrm{Sun} \mathrm{Y,} \mathrm{Grabowski} \mathrm{GA.} \mathrm{Multi-system} \mathrm{disorders} \mathrm{of}$ glycosphingolipid and ganglioside metabolism. J Lipid Res 2010; 51(07):1643-1675

69 Muir R, Ballan J, Clifford B, et al. Modelling maternal obesity: the effects of a chronic high-fat, high-cholesterol diet on uterine expression of contractile-associated proteins and ex vivo contractile activity during labour in the rat. Clin Sci (Lond) 2016;130(03):183-192

70 Boyle KE, Patinkin ZW, Shapiro ALB, et al. Maternal obesity alters fatty acid oxidation, AMPK activity, and associated DNA methylation in mesenchymal stem cells from human infants. Mol Metab 2017;6(11):1503-1516

71 Badawy AA. Tryptophan metabolism, disposition and utilization in pregnancy. Biosci Rep 2015;35(05):e00261

72 Ryding EL, Wijma B, Wijma K, Rydhström H. Fear of childbirth during pregnancy may increase the risk of emergency cesarean section. Acta Obstet Gynecol Scand 1998;77(05):542-547

73 Ogunyemi D, Jovanovski A, Liu J, et al. The contribution of untreated and treated anxiety and depression to prenatal, intrapartum, and neonatal outcomes. AJP Rep 2018;8(03):e146-e157

74 Huang J, Li HJ, Wang J, et al. Prenatal emotion management improves obstetric outcomes: a randomized control study. Int J Clin Exp Med 2015;8(06):9667-9675

75 Zhao Y, Munro-Kramer ML, Shi S, Wang J, Luo J. A randomized controlled trial: effects of a prenatal depression intervention on perinatal outcomes among Chinese high-risk pregnant women with medically defined complications. Arch Women Ment Health 2017;20(02):333-344

76 Cordeaux Y, Pasupathy D, Bacon J, Charnock-Jones DS, Smith GC. Characterization of serotonin receptors in pregnant human myometrium. J Pharmacol Exp Ther 2009;328(03):682-691

77 Horgan RP, Clancy OH, Myers JE, Baker PN. An overview of proteomic and metabolomic technologies and their application to pregnancy research. BJOG 2009;116(02):173-181

78 Bahado-Singh RO, Akolekar R, Mandal R, et al. First-trimester metabolomic detection of late-onset preeclampsia. Am J Obstet Gynecol 2013;208(01):58.e1-58.e7

79 Heazell AE, Brown M, Worton SA, Dunn WB. Review: The effects of oxygen on normal and pre-eclamptic placental tissue-insights from metabolomics. Placenta 2011;32(Suppl 2):S119-S124

80 Bahado-Singh RO, Akolekar R, Mandal R, et al. Metabolomics and first-trimester prediction of early-onset preeclampsia. J Matern Fetal Neonatal Med 2012;25(10):1840-1847
81 Heazell AE, Brown M, Dunn WB, et al. Analysis of the metabolic footprint and tissue metabolome of placental villous explants cultured at different oxygen tensions reveals novel redox biomarkers. Placenta 2008;29(08):691-698

82 Horgan RP, Broadhurst DI, Dunn WB, et al. Changes in the metabolic footprint of placental explant-conditioned medium cultured in different oxygen tensions from placentas of small for gestational age and normal pregnancies. Placenta 2010;31 (10):893-901

83 Powell KL, Carrozzi A, Stephens AS, et al. Utility of metabolic profiling of serum in the diagnosis of pregnancy complications. Placenta 2018;66:65-73

84 Kelly RS, Giorgio RT, Chawes BL, et al. Applications of metabolomics in the study and management of preeclampsia; a review of the literature. Metabolomics 2017;13(07):86

85 Kadakia R, Scholtens DM, Rouleau GW, et al. Cord blood metabolites associated with newborn adiposity and hyperinsulinemia. J Pediatr 2018;203:144-149.e1

86 Caboni P, Meloni A, Lussu M, et al. Urinary metabolomics of pregnant women at term: a combined GC/MS and NMR approach. J Matern Fetal Neonatal Med 2014;27(02, Suppl 2):4-12

87 Meloni A, Palmas F, Barberini L, et al. PROM and labour effects on urinary metabolome: a pilot study. Dis Markers 2018; 2018:1042479

88 Virgiliou C, Gika HG, Witting M, et al. Amniotic fluid and maternal serum metabolic signatures in the second trimester associated with preterm delivery. J Proteome Res 2017;16(02):898-910

89 Bafor EE, Rowan EG, Edrada-Ebel R. Toward understanding myometrial regulation: metabolomic investigation reveals new pathways of oxytocin and ritodrine activity on the myometrium. Reprod Sci 2017;24(05):691-705

90 Zaki MN, Hibbard JU, Kominiarek MA. Contemporary labor patterns and maternal age. Obstet Gynecol 2013;122(05):1018-1024

91 Harper LM, Caughey AB, Odibo AO, Roehl KA, Zhao Q, Cahill AG. Normal progress of induced labor. Obstet Gynecol 2012;119(06): $1113-1118$

92 Min CJ, Ehrenthal DB, Strobino DM. Investigating racial differences in risk factors for primary cesarean delivery. Am J Obstet Gynecol 2015;212(06):814.e1-814.e14

93 Creanga AA, Syverson C, Seed K, Callaghan WM. Pregnancyrelated mortality in the United States, 2011-2013. Obstet Gynecol 2017;130(02):366-373

94 Metcalfe A, Wick J, Ronksley P. Racial disparities in comorbidity and severe maternal morbidity/mortality in the United States: an analysis of temporal trends. Acta Obstet Gynecol Scand 2017

95 Patel MJ, Batch BC, Svetkey LP, et al. Race and sex differences in small-molecule metabolites and metabolic hormones in overweight and obese adults. OMICS 2013;17(12):627-635

96 Hill M, Reed KL, Cohen WR. Oxytocin utilization for labor induction in obese and lean women. J Perinat Med 2014

97 Walsh J, Foley M, O'Herlihy C. Dystocia correlates with body mass index in both spontaneous and induced nulliparous labors. J Matern Fetal Neonatal Med 2011;24(06):817-821 